\title{
Expression levels of HER2/neu and those of collocated genes at 17q12-21, in breast cancer
}

\author{
RONAN W. GLYNN ${ }^{1}$, NICOLA MILLER ${ }^{1}$, SARAH MAHON $^{2}$ and MICHAEL J. KERIN ${ }^{1}$ \\ ${ }^{1}$ Department of Surgery and ${ }^{2}$ Division of Pathology, Clinical Science Institute, \\ National University of Ireland, Galway, Costello Road, Galway, Ireland
}

Received February 24, 2012; Accepted March 26, 2012

DOI: $10.3892 /$ or.2012.1780

\begin{abstract}
HER2/neu is associated with poorer clinical outcome in breast cancer. Expression patterns of co-localised cancerassociated genes at 17q12-21 were examined using RT-PCR. The study group consisted of a 96-patient cohort. Relative quantity of mRNA expression was calculated using the comparative cycle threshold method and Qbase software. Results were analysed to detect expression patterns among the genes, and to identify associations between expression levels and clinical data. Levels of HER2/neu correlated with those of $G R B 7(\mathrm{r}=0.551, \mathrm{p}<0.001)$, RARA ( $\mathrm{r}=0.391, \mathrm{p}<0.001), R P L 19(\mathrm{r}=0.549, \mathrm{p}<0.001)$ and LASPI ( $\mathrm{r}=0.399, \mathrm{p}<0.001)$. GRB7 was significantly inversely associated with improved DFS at 60 months ( $\mathrm{p}=0.036) . R A R A$ levels were greater in HER2/neu-positive as opposed to HER2/ neu-negative patients $(\mathrm{p}=0.021)$; levels were significantly higher in ER-positive patients, relative to those who were ER-negative ( $\mathrm{p}=0.003$ ). Levels of RPL19 were significantly higher in the HER2/neu-overexpressing $(\mathrm{p}=0.010)$ and luminal B subtypes $(\mathrm{p}=0.007)$. LASP1 levels were higher in those patients who had been classified clinically as HER2/neu-positive $(\mathrm{p}=0.004)$. This study reaffirms the correlation between HER2/neu and the co-localised LASPI and GRB7; the latter target may hold additional significance in addition to being a surrogate marker for HER2/neu expression. The relationship identified between $R A R A$ and ER-positivity may herald an avenue for targeted therapy of these tumours.
\end{abstract}

\section{Introduction}

The molecular taxonomy of breast cancer, as first described by Perou et al (1), has revolutionised how clinicians approach and manage breast cancer. Our treatment options have not, as yet, evolved to the point where each subtype can be considered

Correspondence to: Dr Nicola Miller, Department of Surgery, Clinical Science Institute, National University of Ireland, Galway, Costello Road, Galway, Ireland

E-mail: nicolla.miller@nuigalway.ie

Key words: GRB7, HER2/neu, LASP1, RARA, RPL19, TUBG1 entirely separate, however. In particular, numerous questions remain in relation to HER2/neu function, its influence on tumourigenesis and cancer progression and, most importantly from a clinical standpoint, the underlying mechanisms through which it impacts on our treatment strategies. One approach to answering these questions has been to focus on the area of chromosome 17 in which HER2/neu is located, 17q12-21 (the HER2/ neu amplicon), in order to identify other genes in the vicinity.

Growth factor receptor-bound protein 7 (GRB7) forms part of the GRB7 family of proteins, along with GRB10 and GRB14, and it acts as an adaptor molecule. Numerous binding partners have been identified for GRB7, including HER2/neu (2). GRB7 has been demonstrated to be coamplified with HER2/neu $(2,3)$ in breast tumour biopsies. In addition, it is included on Oncotype DX, the commercially available multigene molecular assay which can provide individualised risk estimates for patients with breast cancer, based on the expression levels of 16 cancer-related genes in reference to 5 invariant genes (4).

Other co-located targets of interest include retinoic acid receptor $\alpha(R A R A)$, an estrogen-regulated gene with potential as a target for cancer prevention and therapy because treatment with retinoids leads to cell growth arrest and apoptosis in ER-positive breast cancer cells $(5,6)$, and ribosomal protein 19 (RPL19) a member of a family comprising $>70$ different proteins that form the large and small ribosomal subunits. Whilst protein expression of RPL19 was first demonstrated to correlate with that of HER2/neu in 1993 (7), and in 2003 it was shown to be co-expressed with HER2/neu on a cDNA microarray (8), its expression has not yet been examined by RT-PCR (real-time polymerase chain reaction), and its association with clinicopathological variables or outcome in breast cancer remains to be elucidated.

Given that many of the chemotherapeutic agents currently in use target the microtubule, including the microtubulestabilising agents, paclitaxel docetaxel and the epithilones A and $\mathrm{B}$, and the microtubule-destabilising agents, vincristine and vinblastine, $\gamma$-tubulin is potentially a target in cancer chemotherapy, $\gamma$-tubulin 1 (TUBGI) was the first $\gamma$-tubulin gene to be discovered in 1991 (9), and its upregulation has been postulated to facilitate rapid division of tumour cells (10). Interestingly, in some preclinical studies, taxane resistance has been reversed with the use of trastuzumab in HER2/neu-positive tumours (11), and the relationship between HER2/neu and TUBG1 certainly warrants further consideration. 
One final target of interest is LIM and SH3 domain protein 1 $(L A S P 1)$. Originally identified in a cDNA library of metastatic breast cancer (12), the cellular function of LASP1 remains unknown. Knockdown of $L A S P I$ in the breast cancer cell lines BT-20 and MCF-7, and the ovarian cancer cell line SKOV-3 has however been demonstrated to result in strong inhibition of proliferation and migration $(13,14)$. In 2006, LASPI was identified as one of 5 genes down-regulated in poor prognosis, lymph-node-positive breast cancer (15). LASPI has also been identified as a potential p53 (a tumour suppressor) target in hepatocellular carcinoma, with further work suggesting that p53 may play a role in influencing tumour metastasis through LASPI (16).

The primary aim of this work was to further characterise, using RT-PCR, the relationship between HER 2/neu and the aforementioned candidate genes (GRB7, RARA, RPL19, TUBG1 and LASPI) located at the HER2/neu amplicon on chromosome 17, thus identifying targets with prognostic or predictive significance in the clinical management of breast cancer.

\section{Materials and methods}

The study group for RT-PCR consisted of a 96-patient cohort (Table I); mean patient age at diagnosis of breast cancer was 57.6 years (SD 13.3, range 28-92), and the median period of follow-up was 49 months (range 1-224) (Table I). HER2/neu status had been previously determined by scoring membranous staining according to the HercepTest ${ }^{\mathrm{TM}}$ (Dako) protocol. Similarly, routine clinical determination of ER and PR status had been performed at Galway University Hospital using the Allred scoring method (17). Patients were classified according to tumour characteristics as follows: luminal A (estrogen receptor (ER)-positive and/or progesterone receptor (PR)-positive and not HER2/neu-positive); luminal B (ER-positive and/or PR-positive and HER2/neu-positive); HER2/neu overexpressing (HER2/ neu-positive, ER-negative, PR-negative); and basal-like or triple-negative (ER-negative, PR-negative, HER2/neu-negative). According to this classification, 35 (36.5\%) patients in the study group had luminal A breast cancer, 31 (32.3\%) had luminal B, 17 (17.7\%) were HER2/neu overexpresssing, and 13 (13.5\%) were basal-like. Fifty-seven patients $(59.4 \%)$ were ER-positive and 55 (57.3\%) were PR-positive; the PR status for 1 patient was unknown. Forty-eight patients (50.0\%) were HER2/neu-positive, and 48 (50.0\%) were HER2/neu-negative.

$R T-P C R$. Following informed, signed consent, tissue specimens were snap-frozen in liquid nitrogen immediately following surgical excision, and subsequently stored at $-80^{\circ} \mathrm{C}$. Ethical approval from the Galway University Hospital's Research Ethics Committee was granted to store and use this material for translational research programmes. This tissue was later homogenised, and total RNA was extracted. RNA concentration and purity was determined using a NanoDrop spectrophotometer. Aliquots of RNA equivalent to $1 \mu \mathrm{g}$ were reverse transcribed using SuperScript ${ }^{\mathrm{TM}}$ III (Invitrogen). RT-PCR reactions were then carried out in final volumes of $10 \mu \mathrm{l}$ using a 7900HT sequence detection system [Applied Biosystems (AB)], using TaqMan probes, optical 96-well fast plates and sequence detection system (SDS) software (AB). RT-PCR reaction volumes consisted of cDNA $(1 \mu \mathrm{l})$, mastermix $(5 \mu \mathrm{l})$, TaqMan probe mix $(0.5 \mu \mathrm{l})$, and nuclease-free water $(3.5 \mu \mathrm{l})$.
Table I. Study group.

No.

\begin{tabular}{cr}
\hline Grade & \\
0 & 5 \\
1 & 5 \\
2 & 28 \\
3 & 58
\end{tabular}

$\mathrm{T}$

1218

2248

323

4

Unknown

Nodal status

$\begin{array}{ll}0 & 50\end{array}$

1216

2215

3213

Unknown 2

$\mathrm{M}$

0

$1 \quad 16$

Unknown 3

NPI

126

228

3239

4 39

Unknown 4

Stage

1

2

323

$4 \quad 16$

Unknown 3

ER status

$\mathrm{ER}^{+}$

$\mathrm{ER}^{-} \quad 39$

Unknown 0

PR status

$\mathrm{PR}^{+}-55$

$\mathrm{PR}^{-} \quad 40$

Unknown

HER2/neu status

HER2/neu ${ }^{+}$

HER2/neu

Unknown

5
5
8
8
8
8
3
6
1
16
13
13

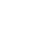

7

6

3

6

8

(

4

7

7

6

3

5

0

5

40

1

48

48
0 
cDNA, synthesised from commercially available breast cancer cell line RNA, was included on each 96-well plate as an interassay control. All reactions were performed in triplicate, and the threshold standard deviation for intra- and inter-assay replicates was 0.3 .

Relative quantity of mRNA expression was calculated using the comparative cycle threshold $(\Delta \Delta \mathrm{Ct})$ method and Qbase software. The geometric mean of the cycle threshold value of the endogenous control genes PPIA and MRPL19 was used to normalize the data, and the lowest expressed sample was used as a calibrator. Relative quantities were logarithmized $(\log 10)$, and distribution of the data set was assessed using the KolmogorovSmirnov test in SPSS version 15.0. Logarithmized values did not vary significantly from a normal distribution. Statistical tests employed to assess the data included Pearson's correlation coefficient, Student's t-test and one-way ANOVA for between groups analysis. Kaplan-Meier curves and the log-rank test were employed to investigate for significant relationships between gene amplification and disease-free and overall survival.

\section{Results}

HER2/neu. Gene expression levels of HER2/neu correlated with those of GRB7 (r=0.551, p<0.001), RARA (r=0.391, p<0.001), RPL19 ( $\mathrm{r}=0.549, \mathrm{p}<0.001)$, and LASP1 ( $\mathrm{r}=0.399, \mathrm{p}<0.001)$. HER2/neu expresssion did not correlate with that of TUBG1 $(\mathrm{r}=0.173, \mathrm{p}=0.091)$. Expression levels of HER2/neu were significantly higher in those patients who had been classified clinically as HER2/neu-positive using immunohistochemistry (IHC) and fluorescent in situ hybridisation (FISH) $(\mathrm{p}<0.001)$. Levels did not differ significantly between patients according to either their ER $(\mathrm{p}=0.481)$ or PR status $(\mathrm{p}=0.935)$.

Post hoc analysis using bonferroni adjustment demonstrated that levels of HER2/neu were significantly higher in the HER2/ neu overexpressing and luminal B subgroups relative to levels in both the luminal $A(p<0.001)$ and basal $(\mathrm{p}<0.001)$ subtypes. Gene expression levels of HER2/neu were significantly lower in patients who were $\mathrm{N} 0$ at diagnosis versus those who were $\mathrm{N} 3$ at diagnosis $(p=0.007)$. No differences were seen in amplification levels of HER2/neu according to menopausal status $(\mathrm{p}=0.520)$, tumour grade $(\mathrm{p}=0.634)$.

Levels of HER2/neu were found to be significantly higher in those patients classified as NPI 4 versus those with an NPI of $3(\mathrm{p}=0.002)$. Levels were also higher in the excellent prognostic group (NPI 1), but this did not reach statistical significance. Gene expression of HER2/neu were found to be significantly less in stage $1(\mathrm{p}=0.018)$ and $2(\mathrm{p}<0.001)$ disease when compared to levels in patients with stage 3 disease.

Analysis of the relationship between levels of HER2/neu and disease-free (DFS) $(\mathrm{p}=0.651)$ and overall survival (OS) $(p=0.505)$ using Kaplan-Meier curves failed to demonstrate significant relationships. No differences were noted when we looked at levels in relation to DFS $(\mathrm{p}=0.915)$ and OS $(\mathrm{p}=0.495)$ up to the 60-month time-point.

GRB7. Gene expression levels of $G R B 7$ were significantly higher in patients classified as HER2/neu-positive using IHC and FISH, versus those classified as HER2/neu-negative $(\mathrm{p}=0.009)$. Levels were significantly higher in ER-negative versus ER-positive patients $(p=0.036)$, but no differences were noted according to
PR status ( $\mathrm{p}=0.469)$. GRB7 levels were noted to be significantly higher in the luminal B subgroup relative to the luminal A subgroup ( $\mathrm{p}=0.033$ ); no differences were seen across the other intrinsic subtypes. Levels did not differ according to menopausal status $(\mathrm{p}=0.650)$, tumour grade $(\mathrm{p}=0.650)$, nodal status $(p=0.462)$, stage $(p=0.201)$, or NPI $(p=0.734)$. In relation to survival, although no significant differences were demonstrated for our cohort overall, lower levels of $G R B 7$ were significantly associated with improved DFS $(\mathrm{p}=0.036)$ but not OS $(\mathrm{p}=0.497)$ up to 60 months.

$R A R A$. Levels of RARA were greater in HER2/neu-positive as opposed to HER2/neu-negative patients ( $\mathrm{p}=0.021)$. Gene expression was significantly higher in ER-positive patients, relative to those who were ER-negative $(\mathrm{p}=0.003)$. Levels did not differ significantly between PR-positive and PR-negative patients $(\mathrm{p}=0.403)$. Gene expression was significantly lower in the basallike subtype relative to the luminal $\mathrm{A}(\mathrm{p}=0.038)$, luminal $\mathrm{B}$ $(\mathrm{p}=0.005)$ and HER2/neu overexpressing $(\mathrm{p}=0.012)$ subtypes. Levels did not differ significantly between these latter 3 subgroups. No differences were seen for expression levels relative to clinicopathological variables or in relation to either DFS or OS.

LASP1. LASP1 expression levels were significantly higher in those patients who had been classified clinically as HER2/neupositive $(\mathrm{p}=0.004)$. Levels did not differ significantly according to $E R(p=0.428)$ or PR ( $p=0.109)$ status. Gene expression levels were significantly higher in the HER2/neu overexpressing subtype relative to the luminal A group of patients $(p=0.045)$. Levels did not differ according to menopausal status $(\mathrm{p}=0.076)$, grade $(\mathrm{p}=0.325)$, nodal status $(\mathrm{p}=0.248), \mathrm{NPI}(\mathrm{p}=0.887)$, or stage of disease $(\mathrm{p}=0.053)$. Whilst no significant differences were noted for $L A S P 1$ relative to DFS or OS, a trend towards improved DFS, for those with lower levels of $L A S P 1$, at 60 months ( $\mathrm{p}=0.069$ ) was noted.

RPL19. RPL19 expression levels did not differ significantly according to HER2/neu ( $\mathrm{p}=0.377)$, ER ( $\mathrm{p}=0.167)$ or PR status (0.411). Levels were higher in the HER2/neu overexpressing $(\mathrm{p}=0.010)$ and luminal $\mathrm{B}$ subtypes $(\mathrm{p}=0.007)$, relative to the luminal A subtypes. There was no significant difference in the expression levels of RPL19 according to menopausal status $(\mathrm{p}=0.189)$, grade $(\mathrm{p}=0.955)$, nodal status $(\mathrm{p}=0.562)$, NPI $(p=0.102)$ or stage of disease $(p=0.265)$. No differences were noted in relation to either DFS or OS.

TUBG1. Gene expression levels did not differ significantly between HER2/neu-positive and negative patients $(\mathrm{p}=0.453)$, ER-positive and negative patients $(\mathrm{p}=0.496)$, or PR-positive and negative patients $(\mathrm{p}=0.818)$. Furthermore, no differences were noted across the intrinsic subtypes $(\mathrm{p}=0.083)$, in relation to clinicopathological variables or in relation to either DFS or OS.

\section{Discussion}

This work has confirmed the close relationship which exists between gene expresssion levels of HER2/neu and GRB7. Located at the minimal common region of amplification at the HER2/neu amplicon, GRB7 has been repeatedly demonstrated to co-amplify with HER2/neu $(8,18,19)$. Our work has 
demonstrated that $G R B 7$ is expressed at higher levels in patients with ER-negative breast cancer; this has particular relevance given reports by van Agthoven et al which have suggested that $G R B 7$ is associated with tamoxifen resistance and estrogenindependent proliferation of breast cancer cells in vitro (20); the same authors confirmed this association using RT-PCR on breast cancer tissue samples, and concluded that their data supported previously established associations for HER2/neu and GRB7 with endocrine resistance in breast cancer, further adding to the rationale behind the examination of combinations of endocrine therapies with HER2/neu-targeting compounds. Furthermore, they postulated that their cell line data and other experimental evidence, suggests that GRB7 may exert an active role in tamoxifen resistance of breast cancer independently of HER2/ neu (21). GRB7 protein is known to bind strongly to HER2/neu via its $\mathrm{SH} 2$ domain, and it has been previously suggested that coamplification of the two genes is connected with extramucosal tumour invasion in oesophageal cancer (22). Intriguingly, recent work by Nencioni et al has shown that GRB7 is rapidly upregulated in response to HER2/neu inhibition with lapatinib, and may offer one explanation for acquired resistance to this latter agent, with accumulation of $G R B 7$ in response to HER2/neu signalling inhibition resulting in increased breast cancer cell aggressiveness and metastatic disease progression (23).

The relationship between $G R B 7$ and prognosis has been investigated extensively and, as noted earlier, the gene is located on the Oncotype Dx assay (24) used to predict breast cancer recurrence. In addition, Vinatazer et al (25) demonstrated a correlation between higher levels of GRB7 protein expression and lower disease-free and overall survival, a finding also reported by Cobleigh et al (26) and, more recently, by Nadler et al (27). These findings correlate well with the results of the present work which has demonstrated a significant relationship between higher levels of $G R B 7$ and poorer disease-free survival up to 5 years, and taken together suggest that $G R B 7$ holds greater clinical significance than acting simply as a surrogate marker for expression or amplification of HER2/neu.

Arriola et al, using RT-PCR on 27 breast cancer samples, found that $R A R A$ was one of 4 genes significantly more highly expressed in HER2/neu/TOP2A co-amplified versus HER2/neuamplified breast cancers (the other three were $C A S C 3, C D C 6$ and SMARCE1) (28). To our knowledge, however, this is the first work to detail gene expression levels of RARA by RT-PCR across intrinsic subtypes, and between ER- and HER2/neu-positive and -negative breast cancers. In attempting to sub-classify patients beyond the traditional hormone receptor positive/negative and intrinsic subtype classification systems, it is interesting to note that levels of $R A R A$ were significantly positively correlated with those of HER2/neu, and were significantly greater in those patients classified as ER-positive or HER2/neu-positive, relative to their negative counterparts. There has been much success with targeting RARA in acute promyolocytic leukemia, with approximately $80 \%$ of patients achieving remission when treated with all-trans retinoic acid (ATRA), a synthetic derivative of vitamin A. Although Fitzgerald et al, demonstrated that the ER-negative, HER2/neu-positive cell-line SKBR3 was associated with high expression levels of RARA and response to retinoic acid (29), it appears that SKBR3 is an exception, with most of the work in this area concluding that the majority of the ATRAresistant cell-lines are associated with moderate to high levels of the HER2/neu protein (30-32). In addition, functional work has shown that high expression levels of HER2/neu are associated with resistance to ATRA $(30,33)$, but that a synthetic analogue of ATRA, 4-hydroxyphenyl-retinamide (4HPR), can repress HER2/ neu expression, and thereby sensitise cells to ATRA.

The evidence above suggests that HER2/neu amplification negatively impacts on that of $R A R A$, and hence ER-negative, HER2/neu-positive patients tend not to respond to treatment with ATRA. In contrast, however, our results suggest a significant positive relationship between gene expression levels of HER2/ neu and RARA, suggesting that a significant subgroup of HER2/ $n e u$-positive patients do in fact express $R A R A$, and would thus be amenable to treatment with ATRA. Given that our results also suggest a positive relationship with ER-positivity, it seems reasonable to conclude that this group would largely consist of patients from the luminal B subtype, although it should be noted that levels of $R A R A$ were not significantly higher in this subgroup relative to levels for patients in either the luminal A or HER2/neu overexpressing subgroups.

RPL19 expression levels did not correlate with HER2/ neu status, ER status, or with any of the other clinicopathological variables under study. With the exception of three other studies which have demonstrated coexpression with HER2/ neu $(8,34,35)$, no work has reported either positive or negative relationships between expression or amplification of RPL19 and breast cancer, and it would thus appear that this marker is of questionable significance as a predictive or prognostic marker, as least in breast cancer; it has previously been associated with poorer prognosis in both prostate (36) and colorectal cancer (37).

Expression levels of TUBG1 mRNA have been reported by Niu et al, using RT-PCR, to be increased from normal breast tissue, through to atypical ductal hyperplasia, ductal carcinoma in situ (DCIS) and invasive ductal carcinoma (IDC) of the breast, and furthermore these levels were demonstrated to correlate well with protein expression of TUBG1 as measured using IHC (38). Our work here has built on that of Niu et al, but has not demonstrated an association between expression levels of this gene, and those of HER2/neu, by RT-PCR. Furthermore, no association has been found with ER, PR- or HER2/neu status, and no differences were seen across subtypes or in relation to clinicopathological variables.

The association between LASP1 and HER2/neu has been confirmed in a number of studies $(12,35,39)$. The results reported above have reiterated this relationship using RT-PCR, whilst also demonstrating higher levels of this novel gene in the HER2/ neu overexpessing subtype, and in those patients classified as HER2/neu-positive using IHC and FISH. This latter result does conflict with the only other analysis of LASP1 in relation to clinicopathological variables; Grunewald et al reported that protein expression of LASP1, as assessed using IHC, did not correlate with HER2/neu status (40). On the other hand, these authors stated that increased expression levels of LASP1 correlated with increased tumour size and rates of nodal positivity and, whilst neither of these findings were corroborated by our results, we did note a trend towards improved DFS in those patients with lower levels of $L A S P 1$ gene expression.

This work has elucidated the relationship between HER2/ $n e u$, the intrinsic breast cancer subtypes, and a number of novel targets located at the HER2/neu amplicon on chromosome 17. In particular, we have demonstrated that gene expression levels of 
$R A R A$ differ significantly across the subtypes, and our findings suggest that luminal B patients, in particular, might benefit from treatment with ATRA or a similar derivative. In addition, our findings have reiterated the relationships previously described between HER2/neu and LASP1 and GRB7, and suggest that the latter may hold additional prognostic or predictive significance in addition to being a surrogate marker for HER2/neu expression.

\section{Acknowledgements}

This work was supported by a grant from the National Breast Cancer Research Institute (NBCRI) of Ireland. The funders had no role in the study design, in the collection, analysis and interpretation of data, in the writing of the manuscript, nor in the decision to submit the manuscript for publication.

\section{References}

1. Perou CM, Jeffrey SS, van de Rijn M, et al: Distinctive gene expression patterns in human mammary epithelial cells and breast cancers. Proc Natl Acad Sci USA 96: 9212-9217, 1999.

2. Stein D, Wu J, Fuqua SA, et al: The SH2 domain protein GRB-7 is co-amplified, overexpressed and in a tight complex with HER2 in breast cancer. EMBO J 13: 1331-1340, 1994.

3. Tomasetto C, Regnier C, Moog-Lutz C, et al: Identification of four novel human genes amplified and overexpressed in breast carcinoma and localized to the q11-q21.3 region of chromosome 17. Genomics 28: 367-376, 1995.

4. Paik S, Shak S, Tang G, et al: A multigene assay to predict recurrence of tamoxifen-treated, node-negative breast cancer. N Engl J Med 351: 2817-2826, 2004.

5. Laganiere J, Deblois G and Giguere V: Functional genomics identifies a mechanism for estrogen activation of the retinoic acid receptor alpha1 gene in breast cancer cells. Mol Endocrinol 19 1584-1592, 2005.

6. Wang Q, Yang W, Uytingco MS, Christakos S and Wieder R: 1,25-Dihydroxyvitamin D3 and all-trans-retinoic acid sensitize breast cancer cells to chemotherapy-induced cell death. Cancer Res 60: 2040-2048, 2000.

7. Henry JL, Coggin DL and King CR: High-level expression of the ribosomal protein L19 in human breast tumors that overexpress erbB-2. Cancer Res 53: 1403-1408, 1993.

8. Dressman MA, Baras A, Malinowski R, et al: Gene expression profiling detects gene amplification and differentiates tumor types in breast cancer. Cancer Res 63: 2194-2199, 2003.

9. Zheng Y, Jung MK and Oakley BR: Gamma-tubulin is present in Drosophila melanogaster and Homo sapiens and is associated with the centrosome. Cell 65: 817-823, 1991.

10. Wise DO, Krahe R and Oakley BR: The gamma-tubulin gene family in humans. Genomics 67: 164-170, 2000.

11. Yu D, Liu B, Tan M, Li J, Wang SS and Hung MC: Overexpression of c-erbB-2/neu in breast cancer cells confers increased resistance to Taxol via mdr-1-independent mechanisms. Oncogene 13: $1359-1365,1996$.

12. Tomasetto C, Moog-Lutz C, Regnier CH, Schreiber V, Basset $\mathrm{P}$ and Rio MC: Lasp-1 (MLN 50) defines a new LIM protein subfamily characterized by the association of LIM and SH3 domains. FEBS Lett 373: 245-249, 1995.

13. Grunewald TG, Kammerer U, Schulze E, et al: Silencing of LASP-1 influences zyxin localization, inhibits proliferation and reduces migration in breast cancer cells. Exp Cell Res 312: 974-982, 2006

14. Grunewald TG, Kammerer U, Winkler C, et al: Overexpression of LASP-1 mediates migration and proliferation of human ovarian cancer cells and influences zyxin localisation. Br J Cancer 96: 296-305, 2007.

15. Asaka S, Fujimoto T, Akaishi J, Ogawa K and Onda M: Genetic prognostic index influences patient outcome for node-positive breast cancer. Surg Today 36: 793-801, 2006.

16. Wang B, Feng P, Xiao Z and Ren EC: LIM and SH3 protein 1 (Lasp1) is a novel p53 transcriptional target involved in hepatocellular carcinoma. J Hepatol 50: 528-537, 2009.

17. Allred DC, Harvey JM, Berardo M and Clark GM: Prognostic and predictive factors in breast cancer by immunohistochemical analysis. Mod Pathol 11: 155-168, 1998.
18. Perou CM, Sorlie T, Eisen MB, et al: Molecular portraits of human breast tumours. Nature 406: 747-752, 2000.

19. Luoh SW: Amplification and expression of genes from the 17q11 approximately q12 amplicon in breast cancer cells. Cancer Genet Cytogenet 136: 43-47, 2002.

20. van Agthoven T, Veldscholte J, Smid M, et al: Functional identification of genes causing estrogen independence of human breast cancer cells. Breast Cancer Res Treat 114: 23-30, 2009.

21. van Agthoven T, Sieuwerts AM, Meijer-van Gelder ME, et al: Relevance of breast cancer antiestrogen resistance genes in human breast cancer progression and tamoxifen resistance. J Clin Oncol 27: 542-549, 2009.

22. Tanaka S, Mori M, Akiyoshi T, et al: Coexpression of Grb7 with epidermal growth factor receptor or Her2/erbB2 in human advanced esophageal carcinoma. Cancer Res 57: 28-31, 1997.

23. Nencioni A, Cea M, Garuti A, et al: Grb7 upregulation is a molecular adaptation to HER 2 signaling inhibition due to removal of Akt-mediated gene repression. PLoS One 5: e9024, 2010.

24. Paik S: Development and clinical utility of a 21 -gene recurrence score prognostic assay in patients with early breast cancer treated with tamoxifen. Oncologist 12: 631-635, 2007.

25. Vinatzer U, Dampier B, Streubel B, et al: Expression of HER2 and the coamplified genes GRB7 and MLN64 in human breast cancer: quantitative real-time reverse transcription-PCR as a diagnostic alternative to immunohistochemistry and fluorescence in situ hybridization. Clin Cancer Res 11: 8348-8357, 2005.

26. Cobleigh MA, Tabesh B, Bitterman P, et al: Tumor gene expression and prognosis in breast cancer patients with 10 or more positive lymph nodes. Clin Cancer Res 11: 8623-8631, 2005.

27. Nadler Y, Gonzalez AM, Camp RL, Rimm DL, Kluger HM and Kluger Y: Growth factor receptor-bound protein-7 (Grb7) as a prognostic marker and therapeutic target in breast cancer. Ann Oncol 21: 466-473, 2010.

28. Arriola E, Marchio C, Tan DSP, et al: Genomic analysis of the HER2/TOP2A amplicon in breast cancer and breast cancer cell lines. Lab Invest 88: 491-503, 2008.

29. Fitzgerald P, Teng M, Chandraratna RA, Heyman RA and Allegretto EA: Retinoic acid receptor alpha expression correlates with retinoid-induced growth inhibition of human breast cancer cells regardless of estrogen receptor status. Cancer Res 57: 2642-2650, 1997.

30. Tari AM, Lim SJ, Hung MC, Esteva FJ and Lopez-Berestein G: Her2/neu induces all-trans retinoic acid (ATRA) resistance in breast cancer cells. Oncogene 21: 5224-5232, 2002.

31. De Luca LM, Scita G and Takatsuka J: Retinoic acid downregulates growth, fibronectin and RAR alpha in 3T3 cells: Ha-ras blocks this response and RA metabolism. J Cell Physiol 173: 297-300, 1997.

32. Rishi AK, Gerald TM, Shao ZM, et al: Regulation of the human retinoic acid receptor alpha gene in the estrogen receptor negative human breast carcinoma cell lines SKBR-3 and MDA-MB-435. Cancer Res 56: 5246-5252, 1996.

33. Lim SJ, Gutierrez-Puente Y and Tari AM: N-(4-hydroxyphenyl)retinamide selectively increases All-TRANS retinoic acid inhibitory effects in HER2/NEU-overexpressing breast cancer cells. Tumour Biol 23: 279-286, 2002.

34. Bertucci F, Borie N, Ginestier C, et al: Identification and validation of an ERBB2 gene expression signature in breast cancers. Oncogene 23: 2564-2575, 2004.

35. Orsetti B, Nugoli M, Cervera N, et al: Genomic and expression profiling of chromosome 17 in breast cancer reveals complex patterns of alterations and novel candidate genes. Cancer Res 64: 6453-6460, 2004.

36. Bee A, Ke Y, Forootan S, et al: Ribosomal protein 119 is a prognostic marker for human prostate cancer. Clin Cancer Res 12: 2061-2065, 2006.

37. Huang CJ, Chien CC, Yang SH, et al: Faecal ribosomal protein L19 is a genetic prognostic factor for survival in colorectal cancer. J Cell Mol Med 12: 1936-1943, 2008.

38. Niu Y, Liu T, Tse GM, et al: Increased expression of centrosomal alpha, gamma-tubulin in atypical ductal hyperplasia and carcinoma of the breast. Cancer Sci 100: 580-587, 2009.

39. Clark GM, Dressler LG, Owens MA, Pounds G, Oldaker T and McGuire WL: Prediction of relapse or survival in patients with node-negative breast cancer by DNA flow cytometry. N Engl J Med 320: 627-633, 1989.

40. Grunewald TG, Kammerer U, Kapp M, et al: Nuclear localization and cytosolic overexpression of LASP-1 correlates with tumor size and nodal-positivity of human breast carcinoma. BMC Cancer 7: $198,2007$. 